\title{
Estomatitis Asociada a Metotrexato: Un Reto Diagnóstico
}

\author{
Methotrexate Associated Stomatitis: A Diagnostic Challenge
}

\author{
María Luisa Sánchez Romero1; Juan Antonio Cepeda Bravo1; Francisco Javier Tejeda Nava; \\ Soledad Delgado Pastrana ${ }^{3} \&$ Saray Aranda Romo 4
}

\begin{abstract}
SÁNCHEZ, R. M. L.; CEPEDA, B. J. A.; TEJEDA, N. F. J.; DELGADO, P. S. \& ARANDA, R. S. Estomatitis asociada a metotrexato: Un reto diagnóstico. Int. J. Odontostomat., 14(4):572-574, 2020.

RESUMEN: Las úlceras orales (UO) son uno de los signos de toxicidad por metotrexato (Mtx) aún en pacientes con esquemas de dosis bajas para el control de artritis reumatoide (AR). En estos casos establecer un diagnóstico correlacionando UO con el medicamento puede ser un reto. Presentamos 2 casos clínicos de pacientes con AR en tratamiento con Mtx, las cuales desarrollaron UO. En los dos casos, interesantemente los pacientes fueron evaluados tanto por especialistas del área médica y oral sin tener un resultado satisfactorio después de múltiples tratamientos. Las UO resolvieron posterior a la suspensión del medicamento.Se estableció el diagnóstico de Estomatitis por Metotrexato (EMtx) por un especialista en medicina oral. El manejo multidisciplinario en estos casos es clave para el establecimiento de un diagnóstico y tratamiento oportuno.
\end{abstract}

PALABRAS CLAVE: ulceras orales, metotrexato, toxicidad, envejecimiento, artritis reumatoide.

\section{INTRODUCCIÓN}

Se estima que un $6,39 \%$ de la población general sufre algún tipo de enfermedad reumatológica crónica inflamatoria, esta frecuencia se incrementa con la edad (Puig et al., 2019). Muchos de estos pacientes sobre todo adultos mayores podrán presentar manifestaciones orales como primer signo o síntoma de reacciones adversas a fármacos, con gran frecuencia los antirreumáticos modificadores de la enfermedad (FARMES) (Gualtierotti et al., 2018). La principal indicación para el uso de estos fármacos es la AR y el fármaco más ampliamente utilizado a dosis bajas por su efectividad es el Mtx (Cardiel, 2006). Sin embargo se han reportado una gran variedad de reacciones adversas, sobre todo en adultos mayores, la Emtx es la más común (Nogueiras Álvarez et al., 2019). La Emtx se observa en los tejidos con una alta división celular como el epitelio de la mucosa oral (Delyon et al., 2015). Tanto los médicos especialistas en el tratamiento de la AR, como los odontólogos deben identificar los efectos secundarios de este fármaco (Deeming et al., 2005).
La Emtx se manifiesta en el 30 a $80 \%$ de los pacientes y es considerada un signo temprano de toxicidad (Troeltzsch et al., 2013). A pesar de su alta frecuencia, las UO no son diagnosticadas de manera oportuna (Kalantzis et al., 2005), tal vez debido a que las lesiones ulcerativas muestran características clínicas similares a enfermedades inflamatorias, infecciosas o vesiculo-ampollares. Por tal motivo los pacientes son comunmente tratados con antivirales, antibióticos, analgésicos e incluso antimicóticos sin éxito (Troeltzsch et al.). Este tipo de fármacos incrementan la toxicidad del Mtx retardando la cicatrización de las UO y favoreciendo así, aún más la complicación del cuadro (Deeming et al.). La presencia de UO en este grupo de pacientes debe alertar al clínico sobre la toxicidad del Mtx (Deeming et al.). Los médicos son el primer profesional en contacto con el paciente por tal motivo resulta deseable un diagnóstico oportuno mediante la historia clínica completa incluyendo aspectos orales y la evaluación de la mucosa oral debe llevarse a cabo de manera rutinaria (Kalantzis

\footnotetext{
${ }^{1}$ Departamento de Periodoncia, Facultad de Estomatología, Universidad Autónoma de San Luis Potosí, México.

2 Departamento de Imagenología, Facultad de Estomatología, Universidad Autónoma de San Luis Potosí, México.

${ }^{3}$ Práctica privada en Ortodoncia. San Luis Potosí, San Luis Potosí, México.

${ }^{4}$ Clínica de Diagnóstico, Facultad de Estomatología, Universidad Autónoma de San Luis Potosí, México.
} 
et al.). Se sugiere que estos pacientes puedan referirse al médico oral con la finalidad de establecer el diagnóstico y tratamiento de forma multidisciplinaria (Kalantzis et al.). Dicho manejo tendrá un impacto directo en mejorar la calidad de vida del paciente. El propósito de este escrito es presentar 2 casos clínicos de pacientes mayores de 70 años que desarrollaron UO y recibieron previamente múltiples tratamientos sin resolución del cuadro por otros especialistas del área médica y odontológica. Se discute sobre la complejidad de establecer diagnósticos certeros sin el apoyo multidisciplinario entre las diferentes especialidades del área de la salud.

\section{PRESENTACIÓN DE LOS CASOS CLÍNICOS}

Paciente 1. Femenino de 72 años acude a consulta por presentar ardor en la mucosa labial de 5 meses de evolución. Se observó una úlcera en el margen bermellón del labio cubierta por una membrana blanco-amarillenta. Antecedentes de AR de 20 años de evolución en tratamiento con Mtx 2,5 mg 3 veces a la semana desde hace 12 años, pentoxifilina, ácido fólico, calcitriol y diclofenaco, en caso de dolor. La paciente fue tratada por diversos médicos con antibióticos, antifúngicos y antivirales sin resolución del cuadro. Al acudir a nuestro servicio, mediante la anamnesis y exploración clínica se estableció el diagnóstico de Emtx. Se lleva a cabo la interconsulta con su médico tratante y se decide la suspensión del medicamento. La úlcera cicatrizó en dos semanas. La paciente, actualmente está medicada con celebrex y gabapentina para el control de la AR y tras 1 año de seguimiento no se han vuelto a presentar UO.

Paciente 2. Femenino de 82 años con UO múltiples mayores bilaterales en mucosa yugal cubiertas por una membrana blanco-amarillenta (Fig. 1), dolor a la masticación y deglución de un año de evolución, lo cual la ha debilitado considerablemente ya que no se ha podido alimentar adecuadamente. Antecedentes de AR 30 años de evolución, diabetes mellitus tipo II, hipotiroidismo e hipertensión arterial. En tratamiento con ledertrexate $2,5 \mathrm{mg}$ de lunes a viernes desde hace 2 años, metformina, levotiroxina, aspirina protect, metoprolol, enalapril, pravastatina, risendronato y caltrate. Multitratada por especialistas del área médica con antibióticos, antimicótico y antivirales sin resolución del caso. Las UO resolvieron en un lapso de 3 semanas posterior a la suspensión del medicamento previa interconsulta con su médico tratante (Fig. 2), se mantiene asintomática desde hace 2 años.

\section{DISCUSIÓN}

El uso de dosis bajas de Mtx para el tratamiento de AR, es el esquema más utilizado por reumatólogos especialmente en Ámérica del Norte (Cardiel). Sin embargo, los efectos adversos a corto y largo plazo se han reportado previamente los cuales incluyen: inflama-

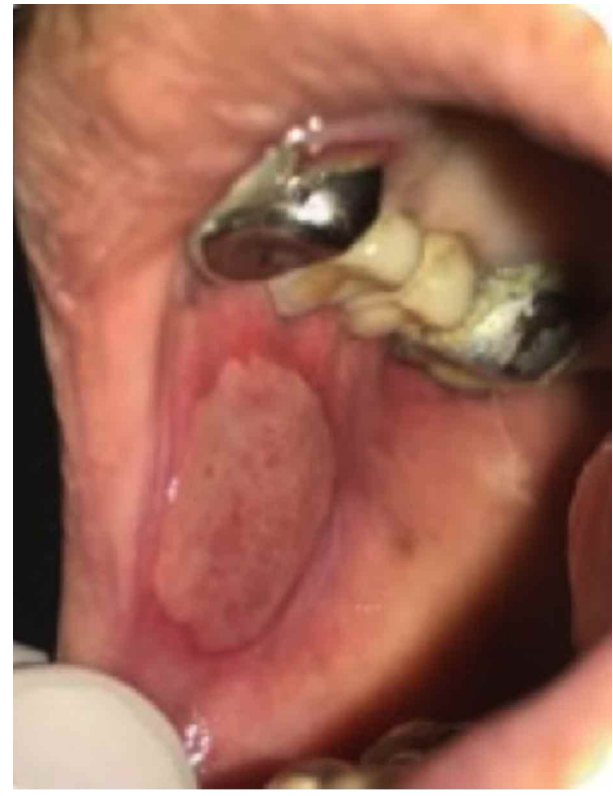

Fig. 1. Ulceración de 1 año de evolución en mucosa yugal.

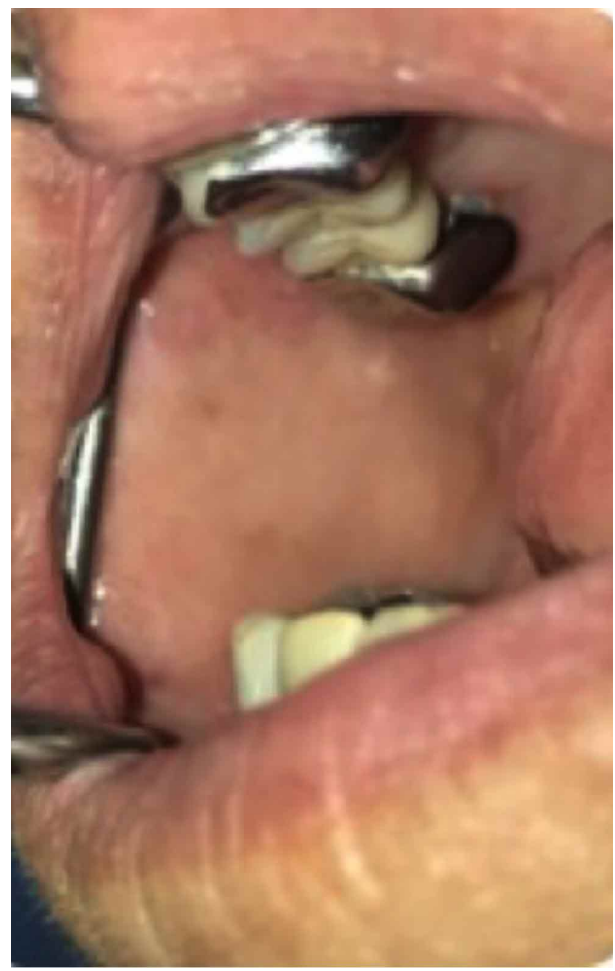

Fig. 2. Remisión de lesión ulcerativa posterior a la suspensión del metotrexato.

ción y ulceración de la mucosa oral, la garganta y una serie de signos y síntomas sistémicos (Troeltzsch et al.; Pivovarov \& Zipursky, 2019). Es clara la relación que existe entre el Mtx y la 
SÁNCHEZ, R. M. L.; CEPEDA, B. J. A.; TEJEDA, N. F. J.; DELGADO, P. S. \& ARANDA, R. S. Estomatitis asociada a metotrexato: Un reto diagnóstico. Int. J. Odontostomat., 14(4):572-574, 2020.

toxicidad en la mucosa oral por lo que la presencia deUO es detectada con una alta frecuencia (64\%); sin embargo para los profesionales de la salud representan un reto diagnóstico (Troeltzsch et al.). En este sentido, en los casos reportados, las pacientes fueron multitratadas previamente por diversos especialistas tanto del área médica como odontológica sin éxito en la resolución del padecimiento, lo cual coincide con lo reportado previamente por otros autores (Deeming et al.; Kalantzis et al.; Troeltzsch et al.). Sin embargo, el tiempo de evolución de las UO antes de establecer el diagnóstico en estos casos fue de 6 meses a 1 años. Estos datos difieren con lo reportado previamente, ya que en promedio el diagnóstico de Emtx se estableció en un período corto de tiempo ( 1 semana a 3 meses) (Deeming et al.). Las UO a pesar de ser autolimitadas, comprometen la calidad de vida del paciente al no poder alimentarse adecuadamente por el dolor. Esta situación exhacerba la deficiencia de nutrientes causando pérdida de peso, llevando asi, a un deterioro de la salud general del paciente e incluso en algunos casos puede llevar a la muerte (Kalantzis et al.). Por tal motivo es imperativo establecer una relación bidireccional médico-odontólogo para el manejo multidisciplinario de estos pacientes. La exploración rutinaria de la mucosa oral con la finalidad de reconocer este primer signo de toxicidad y evitar complicaciones, deben ser prioritarios en estos pacientes. En los casos previamente descritos el diagnóstico se logró establecer por especialistas en medicina oral posterior a 1 año de iniciado el cuadro. La historia clinica resulta la herramienta de diagnóstico crucial para estos pacientes. Si bien en el diagnóstico diferencial de UO se deben considerar otras entidades ulcerativas como las inflamatorias, infecciosas o neoplásicas, observar la resolución de las lesiones en un lapso de 2 a 4 semanas posterior a eliminar el medicamento logra establecer el diagnóstico definitivo. En pacientes con AR en tratamiento con dosis bajas de Mtx que desarrollan UO se debe sospechar de una toxicidad y establecer de manera inmediata el diagnóstico y tratamiento adecuado.

\section{CONCLUSIÓN}

La EMtx es un problema muy frecuente, se debe instaurar un manejo multidisciplinario para estos pacientes con la finalidad de llevar a cabo un diagnóstico y tratamiento adecuado.

AGRADECIMIENTOS. Q.F.B Virginia Flores Gutierrez por su ayuda en el seguimiento de los casos.
SÁNCHEZ, R. M. L.; CEPEDA, B. J. A.; TEJEDA, N. F. J.; DELGADO, P. S. \& ARANDA, R. S. Methotrexate associated stomatitis: A diagnostic challenge. Int. J. Odontostomat., 14(4):572-574, 2020.

ABSTRACT: Oral ulcers (OU) are a sign of methotrexate (Mtx) toxicity, even in patients with rheumatoid arthritis (RA) that are under a low-dose regime. In those cases, establishing a diagnosis correlating $\mathrm{OU}$ with the medication can be quite a challenge. Here we present 2 clinical cases of RA patients under Mtx treatment that developed OU. Interestingly, in both cases the patients were evaluated by two specialists in the medical and dentistry area, and following multiple treatments there was no satisfactory result. However, oral ulcers resolved after stopping the treatment. A diagnosis of Metotrexato stomatitis was established (SMtx) by a specialist in oral medicine. Multidisciplinary management in these cases is key for the establishment of an opportune diagnosis and treatment.

KEY WORDS: oral ulcers, methotrexate, toxicity, elderly, rheumatoid arthritis.

\section{REFERENCIAS BIBLIOGRÁFICAS}

Cardiel, M. H. Latin American Rheumatology Associations of the PanAmerican League of Associations for Rheumatology (PANLAR) \& Grupo Latinoamericano de Estudio de Artritis Reumatoide (GLADAR). First Latin American position paper on the pharmacological treatment of rheumatoid arthritis. Rheumatology (Oxford), 45 Suppl. 2:ii7-22, 2006.

Deeming, G. M. J.; Collingwood, J. \& Pemberton, M. N. Methotrexate and oral ulceration. Br. Dent. J., 198(29):83-5, 2005.

Delyon, J.; Ortonne, N.; Benayoun, E.; Moroch, J.; Wolkenstein, P.; Sbidian, E. \& Chosidow, O. Low-dose methotrexate-induced skin toxicity: Keratinocyte dystrophy as a histologic marker. J. Am. Acad. Dermatol., 73(3):484-90, 2015

Gualtierotti, R.; Marzano, A. V.; Spadari, F. \& Cugno, M. Main oral manifestations in immune-mediated and inflammatory rheumatic diseases. J. Clin. Med., 8(1):21, 2018.

Kalantzis, A.; Marshman, Z.; Falconer, D. T.; Morgan, P. R. \& Odell, E. W. Oral effects of low-dose methotrexate treatment. Oral Surg. Oral Med. Oral Pathol. Oral Radiol. Endod., 100(1):52-62, 2005.

Nogueiras Álvarez, R.; Nan Nan, D. N.; Olmos Martínez, J. M. \& FernándezAyala Novo, M. Severe methotrexate toxicity in elderly: 7 years experience. Rev. Esp. Geriatr. Gerontol., 54(5):296-9, 2019.

Pivovarov, K. \& Zipursky, J. S. Low-dose methotrexate toxicity. C. M. A. J., 191(15):E423, 2019.

Puig, L.; Ruiz de Morales, J. G.; Dauden, E.; Andreu, J. L.; Cervera, R.; Adán, A.; Marsal, S.; Escobar, C.; Hinojosa, J.; Palau, J.; et al. Prevalence of ten Immune-mediated inflammatory diseases (IMID) in Spain. Rev. Esp. Salud Publica, 93:e201903013, 2019.

Troeltzsch, M.; von Blohn, G.; Kriegelstein, S.; Woodlock, T.; Gassling, V.; Berndt, R. \& Troeltzsch, M. Oral mucositis in patients receiving lowdose methotrexate therapy for rheumatoid arthritis: report of 2 cases and literature review. Oral Surg. Oral Med. Oral Pathol. Oral Radiol., 115(5):e28-33, 2013

Dirección para correspondencia:

Saray Aranda Romo.

Facultad de Estomatología

Universidad Autónoma de San Luis Potosí

Manuel Nava No.2 Zona Universitaria.

C.P. 78000. MÉXICO

Recibido : 10-03-2020

Aceptado: 08-05-2020

Email:sarayaranda@fest.uaslp.mx. 